\title{
Factores determinantes del rendimiento en unidades de producción de lechería familiar
}

\section{Factors determining yield in family dairy production units}

\author{
J oaquín Huitzilihuitl Camacho-Vera ${ }^{a}$, Fernando Cervantes-Escotoa*, María Isabel Palacios-Rangela , \\ Fabián Rosales-Noriegab ${ }^{\text {, J uan Manuel Vargas-Canales }}{ }^{a}$
}

\begin{abstract}
RESUMEN
En México las unidades de producción lechera son altamente heterogéneas en cuanto a las condiciones tecnológicas y agroecológicas en que se desarrollan, lo que influye directamente sobre el rendimiento de la ordeña. Este trabajo evalúa la influencia de variables de tipo económico y tecnológico como la escolaridad del productor, el nivel de tecnificación, el tamaño del hato productivo, la inseminación artificial, la presencia de asesoría técnica y el uso de alimento concentrado, sobre el rendimiento obtenido en unidades familiares de producción de la cuenca de Tecamachalco, Puebla. Para verificar este planteamiento empírico se construyó un modelo lineal múltiple y se llevó a cabo una regresión mediante mínimos cuadrados ordinarios. Los resultados mostraron que, a excepción del nivel de tecnificación, todas las variables contempladas en el modelo tuvieron un efecto positivo y significativo sobre la cantidad de litros producidos por vaca por día. Estos resultados cuestionan en buena medida la política de fomento agropecuario que se ha enfocado a la dotación de equipos y habilitación de infraestructura. Se perciben como necesarias políticas públicas y acciones de auto-organización, dirigidas a la mejora de la alimentación de los hatos, a la generalización de la inseminación artificial y a la garantía de asesoría técnica oportuna y de calidad.
\end{abstract}

PALABRAS CLAVE: Nivel de tecnificación, Tamaño del hato, Inseminación artificial, Asistencia técnica, Lechería.

\begin{abstract}
The technological and agro ecological conditions of dairy production units in Mexico are highly heterogeneous. These conditions directly affect milk yield. An evaluation was done of how milk yield in family production units in the Tecamachalco basin, Puebla, Mexico, is influenced by a series of technological and economic variables: producer education level; technological level; productive herd size; artificial insemination; technical assistance; and concentrated feed use. This empirical proposal was verified by building multiple linear models and running an ordinary least mean squares regression. With the exception of technology level, all the studied variables had a positive and significant effect on milk yield (liters / cow / day). These results call into question current livestock promotion policy, which focuses on providing equipment and building infrastructure. Useful modifications in public policy would address self-organization focused on improving herd feeding practices, making artificial insemination general practice, and guaranteeing timely, quality technical assistance.
\end{abstract}

KEY WORDS: Technology level, Herd size, Artificial insemination, Technical assistance, Dairy farming.

\section{INTRODUCCIÓN}

En México la producción de leche se desarrolla en condiciones tecnológicas, agroecológicas y socioeconómicas heterogéneas, dentro de tres sistemas productivos que pueden diferenciarse geográficamente: intensivo (Comarca Lagunera, Durango, Coahuila y Querétaro), familiar (Jalisco y Aguascalientes) y extensivo de doble propósito (regiones tropicales) $^{(1)}$. A la par de ranchos grandes y modernos, se pueden encontrar también unidades

\footnotetext{
Recibido el 1 de marzo de 2016. Aceptado el 18 de abril de 2016.

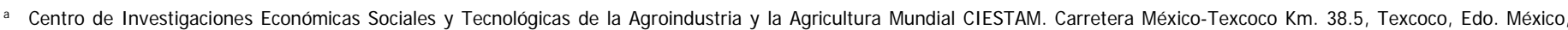
México.

b Unidad Regional de Zonas Áridas. Universidad Autónoma Chapingo. México.

* Autor de correspondencia: tartalian04@gmail.com.
} 
productivas con un nivel de tecnificación menor, cuyos ingresos dependen de la cantidad de unidades animal y no de su productividad(2). Además, existen también unidades familiares, con menor desarrollo tecnológico, pocos vientres en explotación, uso de forrajes de menor contenido proteico y con presencia de componentes tecnológicos promovidos por instituciones gubernamentales (generalmente con instalaciones rústicas) ${ }^{(2)}$.

En este sentido, diversos estudios han relacionado el rendimiento lechero, tanto de explotaciones estabuladas como no estabuladas, con factores biológicos y no biológicos que condicionan la productividad. Dentro de los primeros, la alimentación del ganado y el genotipo destacan por su relevancia para explicar el incremento en rendimiento(3-6). En relación a la mejora genética, se conoce que la utilización de inseminación artificial ha permitido aumentar el rendimiento de los hatos ganaderos a tasas de 1.7 a $2 \%$ anual ${ }^{(7)}$. Por otra parte, variables de tipo socioeconómico y tecnológico también han sido correlacionadas con las diferencias en la productividad lechera. En relación al nivel de tecnificación se han reportado correlaciones positivas con respecto al rendimiento.

En un estudio realizado para unidades familiares de producción de leche mexicanas( ${ }^{(8)}$, se encontró que los cambios en el nivel tecnológico pueden incrementar la productividad entre 26 y $36 \%$ para sistemas extensivos e intensivos respectivamente. En ese mismo estudio, la edad y la escolaridad presentaron también correlaciones positivas con el rendimiento. En otro trabajo realizado por Vélez et $a^{(9)}$ se afirma que la escolaridad del productor está relacionada con una mayor posibilidad de implementar cambios tecnológicos, y con esto, incrementar rendimientos.

La mayoría de las variables de manejo técnico y de tipo socioeconómico están influenciadas por factores territoriales, por lo que pueden ser muy cambiantes de una región a otra. Por tal razón, el presente trabajo tiene como interés fundamental determinar el grado de influencia que ejercen variables de este tipo sobre el rendimiento obtenido por unidades familiares de producción lechera de la cuenca de Tecamachalco, Puebla.
La región de Tecamachalco tiene una larga trayectoria en la producción de leche de bovino que data de finales de la década de los sesentas. En esos años, como resultado la creación del distrito de riego de Valsequillo, se da una reconversión productiva de la región hacia el cultivo de forrajes ${ }^{(10)}$. En virtud de lo anterior y gracias a distintos apoyos gubernamentales, los productores lecheros inician su proceso productivo con ganado de alto registro, producción de forrajes de corte e instalaciones adecuadas. Dada la importancia relativa que el estado de Puebla, y en especial la cuenca de Tecamachalco, han tenido para la producción nacional de leche, esta región se convierte en una buena unidad representativa para el planteamiento de un modelo explicativo de los factores que intervienen en el rendimiento lechero.

\section{MATERIAL Y MÉTODOS}

La región de estudio está conformada por una porción del valle de Tecamachalco ubicada al poniente de la cabecera del municipio de mismo nombre. En este territorio la principal actividad económica es la producción de leche. La superficie agrícola está destinada en su mayoría a la producción de forrajes para la alimentación de los hatos de ganado lechero. Está limitada al oriente por la sierra de Tecamachalco, al poniente por la sierra del Tenzo y al sur por la sierra de Zapotitlán.

La cuenca lechera de Tecamachalco tiene una superficie aproximada de $200 \mathrm{~km}^{2}$ a la cual contribuyen ocho municipios: Tecamachalco, Tochtepec, Atoyatempan, Tlanepantla, Tepeyahualco de Cuauhtémoc, Huitziltepec, Santo Tomas Hueyotlipan y Cuapiaxtla de Madero. Del total de municipios que integran la cuenca, tres tienen un papel destacado por su aporte al total de la producción: Tecamachalco, Tochtepec y Atoyatempan, que aportan más del $70 \%$ del volumen total producido en la región ${ }^{(11)}$.

Para explicar el comportamiento del rendimiento diario de ordeña en litros obtenidos por las explotaciones lecheras de la cuenca, se propuso un modelo lineal múltiple, asumiendo como variables explicativas algunas variables tecnológicas, de competencias del productor y de manejo del hato. 
Este tipo de modelos empíricos permiten identificar la magnitud de la influencia de las variables explicativas sobre la variable de interés. El modelo propuesto quedó expresado de la siguiente manera:

$$
\begin{aligned}
Y=a+ & a_{1} D_{1}+a_{2} D_{2}+a_{3} D_{1} * D_{2}+\beta_{1} X_{1} \\
& +\beta_{2} X_{2}+\beta_{2} X_{3}+\beta_{4} X_{4}+\varepsilon
\end{aligned}
$$

En el cual $X_{1}=$ nivel tecnológico, $X_{2}=$ escolaridad del productor en años, $X_{3}=$ tamaño del hato productivo, $X_{4}=$ años realizando inseminación artificial, $D_{1}=$ presencia de asesoría técnica, $D_{2}=$ uso de alimento concentrado y $D_{1} * D_{2}=$ interacción entre asesoría y uso de alimento.

El planteamiento anterior sigue las recomendaciones de Gujarati y Porter para los modelos de regresión lineal múltiple que consideran más de una variable cualitativa ${ }^{(12)}$. De acuerdo a estos autores, si una variable tiene varias categorías mutuamente excluyentes, el número de variables dicotómicas será igual al número de categorías menos uno. No obstante, cuando se incluye más de una variable dicotómica, es necesario considerar cada variable por separado más el efecto potencial de una interacción. Modelos de regresión múltiple similares han sido usados también para analizar variables de comportamiento social como confianza y capital social ${ }^{(13)}$. El modelo propuesto es similar al utilizado en otros trabajos que plantean modelos lineales para explicar el rendimiento de ganado lechero en función de variables independientes relacionadas principalmente con su genotipo y factores ambientales ${ }^{(3,4,14)}$. En estos casos también se tomaron en cuenta las interacciones entre los factores dicotómicos.

La primera variable del modelo corresponde a un índice tecnológico definido en función de la calidad de la infraestructura y equipamiento existente (características de los corrales, tipo de comederos y equipo de ordeña) ${ }^{(6)}$. Los valores de calificación para cada uno de estos aspectos se sumaron y ese valor fue dividido entre el valor máximo de la suma de los ítems, de esta forma, el valor del índice fluctúa entre cero y uno.

Se consideró también una variable relacionada con el tamaño de la explotación, medido como número de vacas productivas (suma de las vacas en lactancia más las vacas que se encuentran en periodo seco $)^{15}$. En adición a las variables anteriores se consideraron otras dos relacionadas con las características del productor en cuanto a su nivel de competencia. La primera corresponde a escolaridad (medida en años totales de escolaridad) y la segunda (años inseminando) trata de evaluar el impacto de la experiencia en el manejo genético del ganado y del uso de la técnica de inseminación artificial. Finalmente se complementó el modelo con dos variables dicotómicas; a saber, asesoría técnica y uso de alimento concentrado.

Los datos con que se alimentó el modelo se obtuvieron mediante una encuesta estructurada aplicada en 2012 a productores de las principales localidades con actividad económica lechera en el municipio de Tecamachalco: Xochimilco, La Laguna, Colonia Cuauhtémoc, Rubén Jaramillo, Francisco Villa y Santa Rosa. No se dispuso de información confiable sobre el universo de productores con el cual se pudiera obtener una muestra probabilística; por tal razón, se optó por una muestra por conveniencia que no es un método probabilístico, por lo cual las inferencias estadísticas sobre una población deben realizarse con sumo cuidado.

Sin embargo, dado que las unidades de producción se encuentran al lado de los hogares de los productores, y que éstas se ubicaron mediante un "barrido" de las localidades mediante observación directa y por referencia de los mismos productores, se considera que la muestra tiene una alta representatividad. Se obtuvo información de 295 unidades de producción con la que se construyó una base de datos en Excel, que sirvió como base para los tratamientos y estudios posteriores en el software de análisis estadístico Statistical Analysis System.

\section{RESULTADOS Y DISCUSIÓN}

El análisis de los datos muestra un rango amplio en cuanto al tamaño de las unidades lecheras; pudiendo encontrar explotaciones que tienen desde 1 hasta 49 cabezas de ganado. El hato promedio productivo (vacas en producción más vacas secas) fue de $6.55 \pm 6.65$ vacas. Del total de hatos estudiados, en solo cinco se superaron las 30 
unidades en producción, lo que implica que el $98.29 \%$ restante puede ser considerado pequeña producción. Se identificaron productores que tienen 65 años como lecheros, así como también productores de recién entrada con menos de un año en el negocio. En promedio, la antigüedad de los productores en la actividad fue de $14.16 \pm 10.36$ años. El nivel tecnológico calculado para los productores varía desde un mínimo de 0.33 hasta un valor máximo de 1.00 con media de $0.76 \pm 0.156$ (Cuadro 1).

En cuanto al acceso a algún tipo de asesoría técnica, el $60.6 \%$ de los encuestados respondieron de manera negativa a esta pregunta; es decir, sólo $31.4 \%$ tuvieron la posibilidad de acceder a este beneficio. Respecto al uso de alimentos concentrados solo el $57 \%$ lo considera dentro de los piensos diarios dados al ganado, mientras que el $43 \%$ no lo proporciona.

De acuerdo a la prueba de hipótesis realizada con el estadístico $\mathrm{F}$ de Fisher para determinar la significancia global de este modelo, fue significativo al $99 \%(P<0.01)$, lo que indica que por lo menos uno de los parámetros estimados $(\beta \mathrm{i})$ es diferente de cero. Por su parte, el valor del coeficiente de determinación fue de 0.22 , lo que implica que las

Cuadro 1. Valores descriptivos de las principales variables explicativas del modelo

\begin{tabular}{lcccccl}
\hline \multicolumn{1}{c}{ Variable } & N & Mínimo & Máximo & Media & DE & CV \\
\hline Rendimiento, L/día & 293 & 3 & 36 & 14.12 & 5.250 & 37.18 \\
Edad, años & 293 & 14 & 99 & 46.26 & 15.61 & 33.74 \\
Tamaño hato, cabezas & 293 & 1 & 49 & 6.55 & 6.65 & 101.53 \\
Años produciendo & 293 & 0 & 65 & 14.16 & 10.36 & 73.16 \\
Años inseminando & 293 & 0 & 30 & 7.50 & 5.42 & 72.27 \\
Nivel tecnológico & 293 & 0.33 & 1.00 & 0.76 & 0.156 & 20.52 \\
\hline
\end{tabular}

$\mathrm{DE}=$ desviación estándar; $\mathrm{CV}=$ coeficiente de variación.

Cuadro 2. Resumen del modelo de regresión

\begin{tabular}{lccc}
\hline & Parámetro estimado & Error estándar & Valor de t \\
\hline Intercepto & $7.37946^{* *}$ & 1.47004 & 5.02 \\
Nivel tecnológico & 2.54075 & 1.88849 & 1.35 \\
Escolaridad & $0.18850^{*}$ & 0.08305 & 2.27 \\
Tamaño del hato productivo & $0.17147^{* *}$ & 0.04549 & 3.77 \\
Años inseminando & $0.16268^{* *}$ & 0.05347 & 3.04 \\
Tiene asesoría técnica & $1.96613^{* *}$ & 0.79993 & 2.46 \\
Usa alimento concentrado & $2.25361^{* *}$ & 0.67539 & 3.34 \\
Interacción & -1.33974 & 1.19648 & -1.12 \\
Prueba de F & $<0001$ & & \\
$\mathrm{R}^{2}$ & 0.2203 & & \\
\hline
\end{tabular}

* Significativo al 95\% $(P<0.05)$.

** Sianificativo al $99 \%(P<0.01)$. 
variables consideradas como independientes explican un $22 \%$ de la variación del rendimiento. El parámetro estimado para el nivel tecnológico fue el único que resultó no significativo. Todos los demás parámetros estimados resultaron significativos, el de la variable escolaridad a un $95 \%$ de confianza $(P<0.05)$ y el resto al $99 \%(P<0.01)$ (Cuadro 2$)$. El modelo estimado quedó definido de la siguiente forma:

$$
Y=7.37946+1.96613 D_{1}+2.25361 D_{2}-
$$
$1.33974\left(D_{1} D_{2}\right)+2.54075 X_{1}+0.18850 X_{2}+$ $0.17147 X_{3}+0.16268 X_{4}$

Se realizó una prueba gráfica para detectar heteroscedasticidad mediante un gráfico de dispersión, en el cual los valores predichos de la variable dependiente se colocaron en el eje de las abscisas y el cuadrado de los errores estimados $\left(e^{2}\right)$ en el eje de las ordenadas (Figura 1). A pesar de que no se observa ningún patrón específico que indique la presencia de heteroscedasticidad, se realizó una prueba de Glejser para reforzar el análisis.

En el modelo de diagnóstico se tomó como variable dependiente el valor absoluto de los residuales y como variables explicativas las mismas del modelo. La prueba de significancia realizada con el estadístico $\mathrm{F}$ de Fisher mostró que el modelo no es significativo, por lo que no existen evidencias para afirmar que la varianza del error se modifique por efecto de las variables explicativas. Dados estos resultados, se hace evidente que no existen problemas de heteroscedasticidad en el modelo estimado.
En cuanto a la existencia de multicolinealidad, el bajo valor de la $r^{2}$ aunado a la significancia estadística de casi todos los coeficientes de la regresión(12) hace suponer que no existe este problema en el modelo. No obstante, se realizaron regresiones auxiliares de cada variable explicativa con respecto a las demás para determinar el coeficiente de determinación. Una vez obtenida esta información, se asumió la regla práctica de Klein recomendada por Gujarati(12) que sugiere que la multicolinealidad estará presente solo si las $r^{2}$ de las regresiones auxiliares son mayores que la del modelo global. Ninguno de los coeficientes de determinación de las regresiones auxiliares es mayor que el del modelo global, por lo que se argumenta que no existen problemas de multicolinealidad.

Como se esperaba, la escolaridad del productor tiene una relación directamente proporcional con el rendimiento, lo que implica que entre mayor sea el nivel de ésta, el rendimiento lechero es más alto. Este resultado concuerda con lo reportado en otro trabajo(16), en donde se explica la correlación positiva, afirmando que los ganaderos con mayor escolaridad están más dispuestos a integrar nuevas tecnologías que podrían hacer más productivas sus unidades (existe la posibilidad de que la variable educación sea una variable de intermediación que no necesariamente esté asociada de manera directa con la producción, sino que influye sobre otras variables como ingreso, nivel de vida o capacidad de inversión, que a su vez influirían sobre el rendimiento. De esta manera se tendría un efecto indirecto de la escolaridad-educación). Esto sin

Figura 1. Análisis de heteroscedasticidad

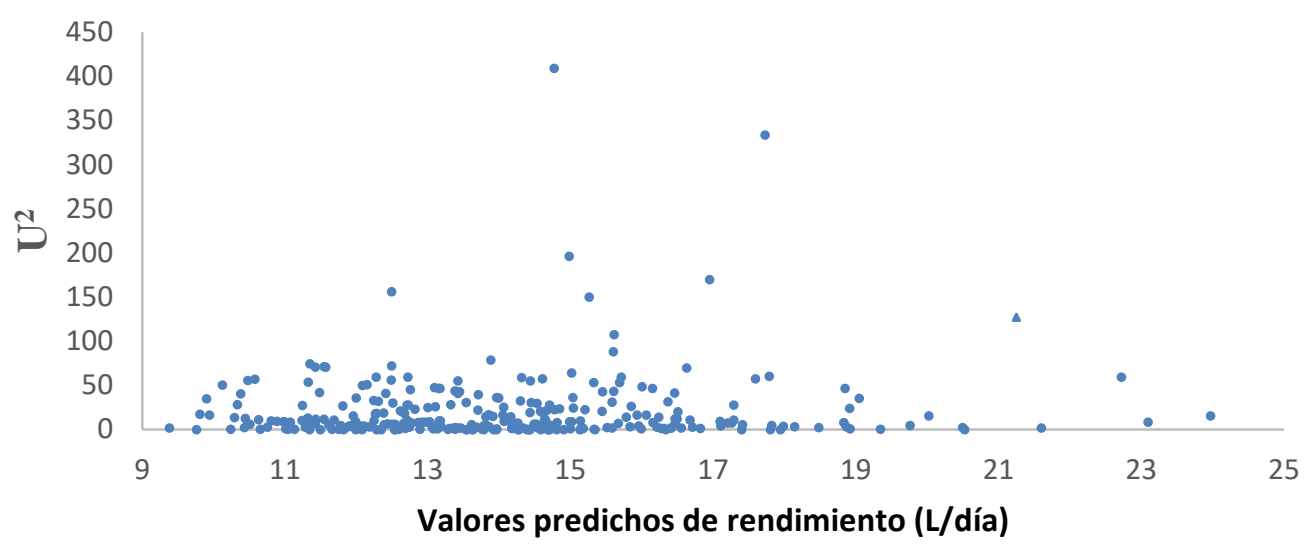


embargo, se contrapone en cierta medida con el nulo efecto del nivel tecnológico en el rendimiento. El coeficiente para este estimador indicaría que, por cada año que aumenta la escolaridad de los productores el rendimiento tiende a incrementarse en 0.19 L por vaca por día.

En cuanto a la relación positiva entre tamaño del hato y rendimiento, puede explicarse por un efecto indirecto derivado de la posibilidad de hacer compras de insumos al mayoreo, que redunda en menores costos unitarios (principalmente de alimento concentrado), lo que potencialmente puede mejorar la cantidad y la calidad de la dieta y con esto el rendimiento en la ordeña(17). El coeficiente de esta variable en el modelo de regresión nos indica que por cada vaca productiva adicional añadida al hato lechero, el rendimiento promedio tendería a incrementarse en $0.17 \mathrm{~L}$.

Una situación similar ocurre con la variable relacionada con el mejoramiento genético del hato lechero. El valor positivo de la pendiente estimada nos indica que conforme aumentan los años realizando esta práctica, la producción promedio en litros por vaca por día se incrementa a razón de 0.16 $\mathrm{L}$ por cada año que se ha realizado inseminación artificial. Este valor representa $1.14 \%$ del rendimiento promedio, lo que se aproxima bastante al valor inferior del rango mencionado en otros estudios como el de Ochoa ${ }^{(7)}$ que va de 1.7 a $2 \%$ de incremento anual en rendimiento derivado de un desarrollo genético del hato.

En cuanto al uso o no de asesoría técnica, el modelo estimado mostró un efecto positivo significativo, en plena correspondencia con el signo esperado para esta variable. Esto implica que la presencia o ausencia de este elemento tiene un impacto directo sobre rendimiento por unidad animal. En el caso del modelo estimado, el valor del parámetro indica que aquellos productores que hacen uso de asesoría técnica tienen un rendimiento superior (casi $2 \mathrm{~L}$ por vaca por día) que aquellos productores que no la utilizan o que no tuvieron acceso a ella.

Con respecto al uso de alimento concentrado en la alimentación del ganado, el efecto reportado para esta variable correspondió con la relación directa esperada, y la magnitud relativa en relación a los efectos de los otros parámetros. El valor estimado expresa que aquellos productores que complementan con alimento concentrado la dieta de su hato ganadero obtienen rendimientos superiores (alrededor de $2.25 \mathrm{~L}$ por vaca por día). Este resultado concuerda con la importancia de la correcta alimentación encontrada en otras investigaciones. La interacción entre el uso de alimento concentrado y la presencia de asesoría técnica no fue significativa; es decir, no existe un efecto aditivo ni multiplicativo sobre el rendimiento por la presencia combinada de estos dos elementos.

Como ya se comentó, de acuerdo a la evidencia obtenida en esta investigación, el nivel tecnológico no mostró un efecto significativo sobre el rendimiento en las unidades productivas de Tecamachalco. Este resultado, contrasta con los resultados de otros estudios para el sector lechero y para otros sectores en los que el nivel tecnológico influye de manera importante sobre los rendimientos.

Las implicaciones prácticas de lo anterior son bastante reveladoras. En los últimos años el grueso de los apoyos destinados al fomento ganadero se ha centrado en el mejoramiento del nivel tecnológico. No obstante, la no significancia de esta variable sobre el rendimiento indica que no debiera ser el factor preponderante de atención en la política agropecuaria diseñada para el sector de bovinos lecheros.

Sería valioso para el sector lechero de la cuenca en cuestión, que se orientaran las acciones de las instituciones de apoyo, de las asociaciones ganaderas y de los productores en lo individual, hacia la mejora de las variables que tienen efecto sobre el rendimiento. Compras de alimento concentrado en colectivo para reducir costos, capacitación de buena calidad para la formulación de dietas, mejoramiento del manejo de la genética del hato, y subsidios en los insumos para inseminación artificial, serían entre otros algunos de los posibles factores sobre los que se tendría que actuar.

Por lo regular, las variables que el modelo ha confirmado relevantes para la productividad de los hatos lecheros han sido menospreciadas o 
francamente olvidadas, tanto por los actores gubernamentales con capacidad de decisión como por los productores. Sería importante que trabajos posteriores indagaran sobre las variables que explican este tipo de comportamiento de productores ante los procesos externos de cambio tecnológico.

\section{CONCLUSIONES E IMPLICACIONES}

Las variables tecnológicas y socioeconómicas seleccionadas en el estudio tienen un efecto positivo y significativo sobre el rendimiento diario de ordeña (exceptuando el nivel tecnológico). La magnitud de la influencia de las variables cuantitativas coincide con lo reportado por otros estudios. Las variables cualitativas tienen un claro efecto de desplazamiento que hace evidente su importancia para explicar la variación del rendimiento lechero. Dadas las características del muestreo es difícil hacer una inferencia estadística sobre el resto de la población de las unidades de producción familiares de la cuenca. No obstante, el tamaño de la muestra, la exhaustividad en su proceso de obtención, la representatividad de las comunidades y la homogeneidad de unidades de producción en cuanto a su tamaño, aportan elementos que robustecen el potencial explicativo del modelo. El bajo valor del coeficiente de determinación corrobora la multideterminación y complejidad del fenómeno, dado que las variables involucradas definen solo una parte del rendimiento. Sin embargo, para el caso específico tratado, la significancia global del modelo y la de las variables independientes le confiere un valor explicativo considerable.

\section{LITERATURA CITADA}

1. García LA, Bottego EM, Quintanal HS. La globalización de la industria lechera mexicana y las empresas agroalimentarias transnacionales. Agroalimentaria 1998;4(7):31-41.

2. Villamar AL, Olivera CE. Situación actual y perspectiva de la producción de leche de bovino en México. Coordinación General de Ganadería. SAGARPA. 2005.

3. Pino T, Martínez GE, Galíndez R, Castejón M, Tovar A. Efecto del grupo racial y algunos factores no genéticos sobre la producción de leche e intervalo entre partos en vacas de doble propósito. Rev Fac Cienc Vet 2011;50(2):93-104.

4. Rodríguez YY, Martínez GE. Efecto de la edad al primer parto, grupo racial y algunos factores ambientales sobre la producción de leche y el primer intervalo entre partos en vacas doble propósito. Rev Fac Cienc Vet 2011;51(2):79-91.

5. Sheen S, Riesco A. Factores que afectan la producción de leche en vacas de doble propósito en trópico húmedo (Pucallpa). Rev Investig Vet Perú 2002; 13(1):25-31.

6. Cervantes F, Cesín A. La pequeña lechería rural o urbana en México, y su papel en el amortiguamiento de la pobreza. Revista Unellez de Ciencia y Tecnología 2009;25: 72-85.

7. Ochoa GP. Mejoramiento genético del ganado bovino productor de leche. En: Enciclopedia bovina. Departamento de genética y bioestadística. UNAM México, DF. 1991.

8. Espinosa JA, Wiggins S, González AT, Aguilar U. Sustentabilidad económica a nivel de empresa: aplicación a unidades familiares de producción de leche en México. Rev Mex Cienc Pecu 2012;42(1):5570.

9. Vélez A, Espinosa JA, Omaña J M, González TA, Quiroz J. Adopción de tecnología en unidades de producción de lechería familiar en Guanajuato, México. Actas Iberoamericanas de Conservación Animal 2013;3:88-96.

10. Camacho JH, Rosales F. Análisis del sistema productivo leche de bovino en el municipio de Tecamachalco, Puebla. En: Bernal $\mathrm{H}$ Ramírez B, coord. Estudios para el desarrollo rural de Puebla. Puebla, México: Colegio de Postgraduados y Altres Costa-Amic Editores; 2014

11. Camacho JH, Aguilar I, Cervantes F. Confianza, normas y participación: Análisis de organizaciones de productores lecheros en México. Arch Zootec 2012;61:197-207.

12. Gujarati D, Porter D. Econometría. 5a ed. México: Editorial Mc. Graw Hill; 2010.

13. Camacho $\mathrm{JH}$. Acciones colectivas y capital social en productores lecheros en la cuenca de Tecamachalco, Puebla. En: Bernal $\mathrm{H}$ Ramírez B, coord. Investigación Interdisciplinaria para el desarrollo rural en Puebla y Tlaxcala. Puebla, México: Colegio de Postgraduados y Altres Costa-Amic Editores; 2011.

14. Abreu O, Labbe S, Rincón EJ, Perozo T. Efecto de algunos factores ambientales sobre la producción de leche en vacas limoneras. Agronomía Trop 1972;22(6):579-586.

15. Cesín A, Fernández M, Ramírez B, Herrera J, Martínez M. Ganadería lechera familiar y producción de queso. Estudio en tres comunidades del municipio de Tetlatlahuca en el estado de Tlaxcala, México. Rev Mex Cienc Pecu 2007;45(1):61-76.

16. Morales PH, Estrada JG, Avilés F, Yong G, López F, Solís AD, Castelán OA. Tipificación de los sistemas campesinos de producción de leche del sur del estado de México. Universidad y Ciencia 2013;29(1):19-31.

17. Vargas B, Cuevas M. Modelo estocástico para estimación de valores económicos de rasgos productivos y funcionales en bovinos lecheros. Agrociencia 2009;43(8):881-893. 